\title{
CONSEQUÊNCIAS DO TRAUMA PÉLVICO EM CÃES
}

\author{
Bernardo Kemper ${ }^{1}$, Ludimila Passos Gonçalves ${ }^{2}$, Michelle Oliveira Vieira ${ }^{2}$, Marcella Luiz \\ Figueiredo ${ }^{3}$, Maíra Santos Severo ${ }^{3}$, Eduardo Alberto Tudury $^{4}$
}

\author{
1 - Departamento de Medicina Veterinária Cirurgia de pequenos animais - UFRPE \\ 2 - Aluna de graduação em medicina veterinária (UFRPE) \\ 3 - Pós-graduanda do PPGCV/UFRPE \\ 4 - Doutor, Professor Associado I, DMV/UFRPE.
}

\begin{abstract}
As fraturas da pelve são comuns e têm recomendação de tratamento conservador ou cirúrgico podendo estar acompanhadas de lesões em estruturas vitais. Objetivouse avaliar as consequências do trauma pélvico em cães e os resultados do tratamento. Foram utilizados 20 animais com fratura na pelve, nos quais a implantação de tratamento, cirúrgico ou conservador, baseou-se no tipo de fratura, extensão das lesões e disponibilidade de recursos. Em 85\% dos animais houve fratura ilíaca, envolvendo estruturas bilaterais em $80 \%$, sendo observados vários transtornos concomitantes como: lesões cutâneas; disquesia; fratura em membros ou de coluna; ruptura de reto, diafragmática ou vias urinárias; trauma crânio encefálico e óbito. Nos operados houve um retorno
\end{abstract}

à deambulação, verificando-se recuperação total após noventa dias. Resultado que não se repetiu nos pacientes tratados conservativamente, sendo observada claudicação persistente, após 90 dias. A estabilização cirúrgica proporcionou redução evidente da dor após sete dias, elevando a qualidade de vida e reduzindo as necessidades de enfermagem, enquanto que $55 \%$ dos não operados vieram a óbito. Dois tiveram que ser operados para corrigir complicações consequentes da falta de correção anterior. Os resultados assinalam que em cães com trauma pélvico deve-se procurar primariamente a resolução cirúrgica e a presença de outras lesões orgânicas concomitantes incapacitantes ou com potencial de induzir ao óbito.

PALAVRAS-CHAVE: cão; fratura; lesões concomitantes; pelve.

\section{CONSEQUENCES OF PELVIC TRAUMA IN DOGS}

Fractures of the pelvis are common and may be accompanied by injuries to vital structures. Conservative treatment or surgery is usually recommended. The aim of this study was to evaluate the consequences of pelvic trauma in dogs and the results of clinical and surgical treatment. Twenty animals with fracture of the pelvis were used. The treatment, surgical or conservative, was primarily based on the type of fracture, extent of injuries and resources availability. $85 \%$ of the animals showed iliac fracture; $80 \%$ of the cases were bilateral pelvic fracture, and concurrent disorders, such as skin lesions, dyschesia, limb and column fracture, colon, urinary tract or diaphragm rupture, head trauma and death, were observed. In patients who underwent surgery there was recovery of ambulation, with full recovery after 90 days. This result was not repeated in patients which underwent 
conservative treatment. The surgical stabilization of bone segments provided clear pain reduction after seven days, increasing life quality and reducing the need for nursing, while $55 \%$ of the animals that were not operated died and other two had to be operated to correct complications as a

KEYWORDS: associated injuries; dog; fractures; pelvis.

\section{INTRODUÇÃO}

Fraturas da pelve são relativamente comuns e correspondem, em clínicas veterinárias, de $20 \%$ a $30 \%$ de todas as fraturas (NEWTON, 1985; OLMSTEAD, 1995; OLMSTEAD \& MATIS, 1998; PIERMATTEI et al., 2006). A maioria compreende fraturas múltiplas nas quais há mais de três ossos envolvidos (OLMSTEAD \& MATIS, 1998; PIERMATTEI et al., 2006).

A causa mais comum de fraturas pélvicas são traumas por acidentes automobilísticos (INNES \& BUTTERWORTH, 1996; OLMSTEAD \& MATIS, 1998; TOMLINSON, 2003; PIERMATTEI et al., 2006); entretanto, elas podem estar associadas a quedas e lesões por arma de fogo (BONATH \& PRIEUR, 1998). São ainda considerados fatores predisponentes doenças metabólicas, doenças ósseas hereditárias e, com menor frequência, tumores ósseos (INNES \& BUTTERWORTH, 1996; BONATH \& PRIEUR, 1998).

As forças que culminam em fraturas pélvicas podem vir de diversos ângulos e ter diferentes intensidades; por essa razão, fraturas pélvicas ocorrem com configurações variadas (OLMSTEAD, 1998). No entanto, como assume a forma de um retângulo, a pelve deve ser fraturada em três diferentes pontos para que haja deslocamento de fragmentos ósseos, sendo as afecções bilaterais comuns (DeCAMP, 1992; JOHNSON, 2007).

$\mathrm{O}$ trauma com energia suficiente para fraturar a pelve normalmente causa danos a outras estruturas esqueléticas e tecido mole e, por isso, um animal com fratura pélvica é frequentemente um paciente politraumatizado (DeCAMP, 1992). Em humanos, a fratura do anel pélvico é comumente associada com significante morbidade e mortalidade. A magnitude dessas fraturas pode variar de lesões menores, causando dor e incômodo, a lesões graves causando morte pré-hospitalar. As fraturas pélvicas podem estar associadas a lesões de vísceras pélvicas e de outras estruturas do organismo. Atenção especial result of the lack of previous surgical correction. The results indicate that, in dogs with pelvic trauma, surgical resolution should be primarily sought, and the presence of other concomitant organic disabling injuries or with the potential to lead to death should be verified.

deve ser dada ao sistema cardiopulmonar, neurológico e urogenital para detectar injúrias como pneumotórax, hérnia diafragmática, ruptura do trato urinário e neuropraxia (HENRY, 1985; DeCAMP, 1992; HOULTON \& DYCE, 1994; HARASEN, 2007).

Para o diagnóstico, a inspeção pode trazer sinais indicativos de fratura pélvica. A sintomatologia depende do grau de trauma, localização da fratura, deslocamento ósseo e dimensão das lesões em tecidos moles (DeCAMP, 1992; BONATH \& PRIEUR, 1998). Após exame clínico geral para estabelecer o estado geral do paciente, um exame ortopédico completo deve ser realizado (OLMSTEAD, 1998). Pela palpação é verificada a simetria pélvica, saúde articular, áreas doloridas ou edemaciadas. Como pontos de orientação, podem ser utilizadas as proeminências ósseas, como asa do ílio, trocanter maior $\mathrm{e}$ tuberosidade isquiática (BONATH \& PRIEUR, 1998). Um exame digital retal adicional cuidadoso pode trazer informações sobre estreitamento do canal pélvico (HENRY, 1985; DeCAMP, 1992; BONATH \& PRIEUR, 1998; OLMSTEAD, 1998; HARASEN, 2007). O diagnóstico definitivo é obtido pelo exame radiográfico. Duas projeções radiográficas, ventro-dorsal e látero-lateral, são necessárias (BONATH \& PRIEUR; 1998; OLMSTEAD, 1998; JOHNSON, 2007). Em alguns casos, são necessárias vistas oblíquas da hemipelve para melhor definição das linhas de fratura e posicionamento dos fragmentos (DeCAMP, 1992; OLMSTEAD, 1995; NEWTON, 1996).

Apesar de o tratamento das fraturas pélvicas poderem ser com repouso e restrição de exercício, o reparo cirúrgico das mesmas geralmente resulta em retorno funcional precoce, com menos dor $\mathrm{e}$ complicação durante a cicatrização. $O$ tratamento conservativo de fraturas pélvicas está indicado quando essas são apresentadas após duas ou mais semanas e quando existem limitações financeiras por parte do proprietário (TOMLINSON, 2003). 
Complicações associadas a fraturas da pelve são usualmente consequências da ausência de redução e fixação das mesmas, podendo resultar em obstipação, constipação crônica e na impossibilidade de ter partos normais na fêmea, decorrente de má união de fratura de corpo de ílio (NEWTON, 1996). Outra complicação associada ao tratamento conservativo de fraturas pélvicas é a claudicação persistente associada a anomalias anatômicas e doença articular degenerativa da articulação coxofemoral (TOMLINSON, 2003).

Uma combinação de técnicas cirúrgicas pode ser necessária para estabilizar a pelve com fraturas múltiplas ou luxação (TOMLINSON, 2003). No tratamento cirúrgico de fraturas pélvicas, maior ênfase é depositada na articulação sacroilíaca, ílio e acetábulo, por se tratar do eixo de transmissão das forças do membro posterior ao esqueleto axial (BONATH \& PRIEUR, 1998). Os animais que sofreram fraturas pélvicas bilaterais se beneficiam do procedimento cirúrgico por serem capazes de voltar a andar com antecipação e por demandarem menor tratamento intensivo (DeCAMP, 1992; JOHNSON, 2007).

Existem diversos métodos de fixação para as fraturas pélvicas que incluem o uso de pinos e fios de Kirschner, placas ortopédicas, parafusos ortopédicos, polimetilmetacrilato (PMMA) e cerclagem interfragmentar (ROEHSIG, 2005; PIERMATTEI, et al., 2006). Além desses, WHITTICK (1978) e KEMPER et al. (2008) descreveram o uso de pinos de Schantz, fixados aos fragmentos ósseos e conectados externamente a barras de Kirchner em fraturas ilíacas.
Os pacientes com fratura de pelve normalmente precisam de analgesia durante os primeiros dias de tratamento. Os antiinflamatórios não esteróidais (AINEs), como o meloxicam e o carprofeno, são geralmente mais adequados, podendo ser associados à opióide se a dor for severa (DeCAMP, 1992; HOULTON \& DYCE, 1994)

Objetivou-se com este trabalho descrever as implicações e ocorrências associadas ao trauma pélvico em cães, em relação ao tipo de fratura, lesões concomitantes e resultado dos tratamentos clínicos e cirúrgicos.

\section{MATERIAL E MÉTODOS}

Neste trabalho, foram utilizados 20 cães com diagnóstico clínico e radiográfico de fratura na pelve, atendidos no Hospital Veterinário (HV) da Universidade Federal Rural de Pernambuco (UFRPE)

Cada paciente passou por uma anamnese detalhada, objetivando-se obter dados relativos à origem e tempo do trauma e à possibilidade da presença de lesões intercorrentes. Após o exame físico, realizou-se o exame ortopédico, com avaliações específicas da região da pelve, além do exame neurológico, transcrevendo-se todas as informações em uma ficha de avaliação idividual.

Utilizaram-se os parâmetros constantes nas Tabelas 1 e 2 para avaliar o grau de claudicação e dor dos pacientes, determinado através da observação da deambulação dos animais em piso de cimento e por meio da palpação para detectar reações dolorosas.

Tabela 1. Parâmetros de avaliação do grau de claudicação utilizados para determinar a situação ortopédica e acompanhar a recuperação dos cães com trauma pélvico.

\begin{tabular}{cl}
\hline Grau & Caracteristicas \\
\hline I & Não usa nem apóia o membro \\
II & $\begin{array}{l}\text { Uso e apoio infrequentes do membro na estação e no caminhar. Não suporta peso } \\
\text { na extremidade afetada, elevando-a ao correr }\end{array}$ \\
III & $\begin{array}{l}\text { Claudicante uso do membro na estação e no caminhar. Parcial suporte do peso na } \\
\text { extremidade, elevando-a ao correr }\end{array}$ \\
IV & $\begin{array}{l}\text { Caminha sem claudicar. Normal na estação. Claudica ao correr sem elevar o } \\
\text { membro }\end{array}$ \\
V & Uso funcional do membro.
\end{tabular}

Fonte: TUDURY \& RAISER (1985). 
Tabela 2. Parâmetros de avaliação do grau de dor utilizados para auxiliar na determinação da situação clínica e acompanhar a recuperação dos cães com trauma pélvico

\begin{tabular}{|c|c|}
\hline Pontuação & Critérios \\
\hline 0 & $\begin{array}{l}\text { Sem dor, sem manifestação de mal estar e sem reação à firme pressão aplicada na } \\
\text { região lesionada }\end{array}$ \\
\hline 1 & Dor leve, sem mal estar, porém com reação à firme pressão; \\
\hline 2 & Dor moderada, com alguns sinais de mal estar, que pioram com a firme pressão; \\
\hline 3 & Dor intensa, com sinais óbvios de mal estar persistente que piora com a firme pressão. \\
\hline
\end{tabular}

Fonte: LASCELLES et al. (1994).

Em sequência, os pacientes foram encaminhados ao setor de radiologia para a realização de exames radiográficos da pelve nas projeções lateral, ventro-dorsal e oblíqua (quando indicado), assim como de outras regiões, para o diagnóstico de injúrias concomitantes.

Além dessas, foram feitas radiografias periódicas nos pacientes, após o atendimento inicial, imediatamente após a cirurgia e nos tempos 30 e 60 dias com o objetivo de avaliar o grau de injúria à pelve, delinear as linhas de fratura, avaliar os implantes ortopédicos, aposição óssea e desenvolvimento de complicação referente ao uso de implante, a cicatrização e o estreitamento do canal pélvico.

Inicialmente, todos os pacientes foram medicados com o antiinflamatório meloxican ${ }^{1}$ $(0,1 \mathrm{mg} / \mathrm{Kg} / \mathrm{uma}$ vez ao dias, durante 7 dias $)$ associado ao opióide cloridrato de tramadol ${ }^{2}$ (2 $\mathrm{mg} / \mathrm{Kg} / \mathrm{de} 8 \mathrm{em} 8$ horas, durante 5 dias) e, nos casos em que houve perda da integridade cutânea, foi acrescido o antibiótico cefalotina ${ }^{3} \quad(25 \mathrm{mg} / \mathrm{Kg} / \mathrm{de} 8$ em 8 horas, durante 7 dias). Dependendo da extensão e do grau de infecção, acrescentou-se enrofloxacina $^{4} \quad(5 \mathrm{mg} / \mathrm{Kg} / \mathrm{de} 12$ em 12 horas, durante 7 dias). Quando presentes, os ferimentos foram higienizados e cobertos com curativos apropriados. Nos pacientes com suspeita de traumatismo nas vias urinária inferiores foi realizada avaliação e manejo com observação de micção, introdução de sondas uretrais e urografias. Ainda foram prescritos laxantes à base de lactulose ${ }^{5}$ $(1 \mathrm{ml} / 4 \mathrm{~kg} /$ por via oral, de 12 em 12 horas) aos pacientes com disquesia.

Foram considerados pertencentes ao grupo plano de tratamento conservador (PTC) pacientes que apresentavam lesões extensas, a ponto de o procedimento cirúrgico ser considerado arriscado, ou quando os pacientes apresentavam fraturas em áreas não envolvidas na transmissão das forças de deambulação, nos pacientes atendidos após mais de 10 dias do trauma, além daqueles para os quais a falta de recursos financeiros representava uma limitação.

Foram considerados pacientes cirúrgicos aqueles com fratura do anel pélvico com uma ou mais das seguintes alterações: marcante redução do canal pélvico; fratura de acetábulo com deslocamento das superfícies articulares; instabilidade pélvica uni ou bilateral, sem risco controlável para realização de procedimento cirúrgico.

Os pacientes foram levados à sala de preparo onde se realizou tricotomia da área a ser operada,

\footnotetext{
1 - Meloxican Merck, Cotia-SP.

2 - Tramaliv ® Teuto, Anápolis-GO.

3 - Cefalotina sódica 1g, InstitutoBioChimico, Ltda,Rio de Janeiro-RJ.

4 - Baytril ${ }^{\circledR} 50$ mg Bayer, São Paulo-SP.

5 - Lactulona ${ }^{\circledR}$, Sankyo, Barueri SP

6 - Clorexidine 2\%. Rioquímica,São José do Rio Preto-SP.
} 
anti-sepsia com clorexidine ${ }^{6} 2 \%$ e administração de cefalotina $^{3}(25 \mathrm{mg} / \mathrm{Kg} / \mathrm{IV})$ acepromazina ${ }^{7} 0,2 \%$ (0.05 $\mathrm{mg} / \mathrm{kg} / \mathrm{IM})$, associada ao cloridrato de tramadol $^{2} \quad(2 \mathrm{mg} / \mathrm{kg} / \mathrm{IM})$. Em seguida, já em ambiente cirúrgico, a veia cefálica foi canulada para infusão de solução fisiológica $(10 \mathrm{ml} / \mathrm{Kg} / \mathrm{IV} /$ hora $)$, indução anestésica com diazepam ${ }^{8}(0,5 \mathrm{mg} / \mathrm{Kg} / \mathrm{IV})$ seguido de propofol $^{9}$ (4 mg/kg/IV), permitindo, assim, intubação. Para anestesia epidural, injetou-se uma associação (1:1) de lidocaína ${ }^{10} \quad 2 \%$ e bupivacaina $^{11} \quad 0,5 \% \quad(1 \mathrm{~mL} / 4,5 \mathrm{~kg})$ no espaço vertebral L7-S1. Em todos os pacientes a manutenção foi feita com oxigênio a $100 \%$ e isofluorano ${ }^{12}$.

Em 10 dos 11 animais submetidos à cirurgia para correção das fraturas da pelve empregou-se a abordagem lateral, conforme descrito por PIERMATTEI \& JOHNSON (2004), através de uma incisão que se estendeu do centro da tuberosidade ilíaca até o trocanter maior do fêmur. O subcutâneo e a fáscia superficial foram deslocados com a pele. Fez-se a seguir uma incisão na fáscia do glúteo superficial, na mesma linha de incisão da pele, permitindo a separação dos músculos tensor da fáscia lata e glúteo médio, alongando entre a incisão desde a face ventral do ílio até a borda cranial do músculo bíceps femoral. Retraindo o músculo glúteo médio dorsalmente, teve-se acesso ao glúteo profundo e a uma porção do corpo do ílio (Figura 1).

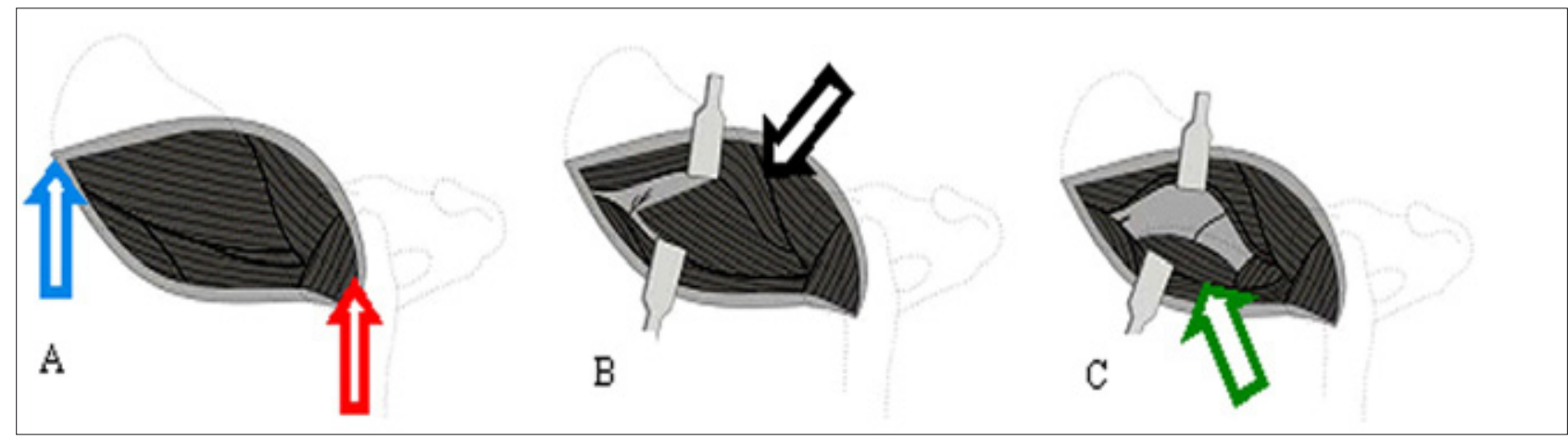

Figura 1 Desenho esquemático mostrando a abordagem cirúrgica ao ílio: A) incisão cutânea iniciando da parte ventral da asa do ílio até o trocanter maior do fêmur (setas azul e vermelha, respectivamente); B) exposição do ílio através da abordagem entre os músculos glúteo médio e tensor da fáscia lata (seta preta); C) elevação dos músculos glúteo médio e profundo e incisão dos músculos glúteos na asa do ílio para exposição adicional (seta verde).

A redução das fraturas foi feita com o auxílio de pinças de redução ósseas (Espanholas e Kern), mantendo os fragmentos reduzidos, permitindo, assim, posicionamento e fixação dos implantes.

Para ação antiinflamatória e analgésica foram utilizados meloxicam ${ }^{13}$ (na dose diária de $0,1 \mathrm{mg} / \mathrm{kg}$, durante sete dias) e cloridrato de tramadol$^{2}$ (na dose de $2 \mathrm{mg} / \mathrm{kg}$, a cada oito horas, durante 5 dias) e antibioticoterapia com cefalexina ${ }^{14}$ (na dose de $25 \mathrm{mg} / \mathrm{kg}$, cada oito horas durante sete dias consecutivos), todos por via oral. Os animais retornaram ao domicilio, após recuperação anestésica, com as orientações a respeito do tratamento medicamentoso, fisioterápico e cuidados de enfermagem.

O curativo da ferida cirúrgica foi realizado com a aplicação de clorexidina ${ }^{15} 1 \%$ duas vezes ao dia, até a retirada dos pontos, sendo também recomendado o uso do colar elisabetano e repouso. A fisioterapia foi preconizada com a aplicação de compressas frias, por 20 minutos, a cada quatro horas durante as primeiras 48 horas, no local da cirurgia; utilização de calor após a fase inicial de inflamação; massagem compressiva para melhora

2 - Tramaliv ${ }^{\circledR}$ Teuto, Anápolis-GO.

3 - Cefalotina sódica 1g, InstitutoBioChimico, Ltda, Rio de Janeiro-RJ.

7 - Acepran ${ }^{\circledR}$ 0,2\%- Univet S. A., 700. São Paulo-SP.

8 - Diazepan 5mg/1mL. Sigma Farma.. Hortolândia-SP.

9 - Diprivan ${ }^{\circledR} 1 \%$. AstraZeneca Cotia Moinho Velho-SP.

10 - Lidovet ${ }^{\circledR}$. Bravet, Rio de Janeiro-RJ.

11 - Marcaína ${ }^{\circledR}$ 0,5\%. AstraZeneca. Cotía Moinho Velho-SP.

12 - Forane. Abbott. São Paulo-SP.

13 - Meloxican - Farmácia de Manipulação

14 - Cefalexina 500mg-Farmácia de manipulação

15 - Merthiolate ${ }^{\circledR}$, Eli Lilly do Brasil Ltda, São Paulo-SP 
do retorno sanguíneo e movimentação passiva dos membros pélvicos com quinze repetições lentas duas vezes por dia. Após sete dias foram iniciados os exercícios ativos incluindo caminhadas leves e natação.

Os animais foram acompanhados clinicamente após a cirurgia por meio de exames específicos, utilizando-se a escala funcional de claudicação, como também os parâmetros de avaliação do grau de dor nos dias 7, 30, 60 e 90. Foi realizada avaliação radiográfica no pós-operatório imediato e nos dias 30 e 60 após a cirurgia.

\section{RESULTADOS E DISCUSSÃO}

Dos 20 cães atendidos, em 11 (55\%) foi realizado tratamento cirúrgico para correção das fraturas do anel pélvico por estarem envolvidas nas fraturas áreas de sustentação como citado por
BONATH \& PRIEUR (1998). Em nove (45\%) instituiu-se tratamento conservativo, sendo a gravidade das lesões, o tempo do atendimento e o tipo de fratura determinante na escolha do tratamento conservativo nesses pacientes, como relatado por PIERMATTEI et al. (2006). Desses, dois foram posteriormente operados para corrigir complicações associadas à ausência de realização de tratamento cirúrgico prévio. Cinco dos não operados vieram a óbito em consequência da gravidade das lesões e dois deles foram tratados conservativamente.

A principal causa das fraturas pélvicas nos animais do experimento foi o acidente automobilístico, 17/20 (85\%), quando comparada com aquelas associadas ao trauma por queda 3/20 (15\%). Este resultado (Tabelas 3 e 4) está de acordo com o citado por outros autores EDWARDS (1993); PAYNE (1993) e TOMLISON (2003).

Tabela 3. Informações dos pacientes submetidos ao tratamento cirúrgico, contendo resenha, etiologia, osso e tipo de fratura e método de fixação utilizado

\begin{tabular}{|c|c|c|c|}
\hline Dados dos Pacientes & $\begin{array}{l}\text { Causa da } \\
\text { Fratura }\end{array}$ & Osso/Tipo de fratura & Método de fixação \\
\hline $\begin{array}{l}\text { No01, macho, Dash, } 1 \text { ano } \\
\text { e } 3 \text { meses, } 8 \mathrm{Kg}\end{array}$ & A. A. & Fratura de ilio esquerdo e bilateral de isquio & Placa com parafusos no corpo do ilio. \\
\hline $\begin{array}{l}\text { No } 02 \text {, macho, Dash } 1 \text { ano } \\
\text { e } 2 \text { meses, } 2,85 \mathrm{Kg}\end{array}$ & A. A. & $\begin{array}{l}\text { Fratura transversa do púbis, do ilio esquerdo e do } \\
\text { acetábulo caudal }\end{array}$ & Parafuso com PMMA no corpo do tho \\
\hline $\begin{array}{l}\mathrm{N}^{\circ} 03, \text { macho, SRD, } 3 \\
\text { anos, } 7 \mathrm{Kg}\end{array}$ & A. A. & $\begin{array}{l}\text { Fratura de corpo de ilio direito, ramo acetabular do } \\
\text { púbis e isquio esquerdo. }\end{array}$ & Parafuso com PMMA no corpo do itio \\
\hline $\begin{array}{l}\mathrm{N}^{\circ} 04, \text { fëmea, Poodle, } \\
\text { lano e } 3 \text { meses, } 5,3 \mathrm{Kg}\end{array}$ & A. A. & Fratura obliqua de ilio esquerdo, isquio e púbis. & Placa com parafusos no corpo do itio. \\
\hline $\begin{array}{l}\text { No 05, fêmea, SRD, } 1 \text { ano, } \\
\qquad 4,5 \mathrm{Kg}\end{array}$ & A. A. & Fratura bilateral de ilio e fêmur distal & $\begin{array}{l}\text { Parafusos com PMMA no corpo do ilio } \\
\text { bilateralmente e osteossintese de fêmur pela } \\
\text { técnica de pino cruzado }\end{array}$ \\
\hline $\begin{array}{l}\text { No } 06, \text { macho, Dash, } 3 \\
\text { anos, } 7,3 \mathrm{Kg}\end{array}$ & A. A. & $\begin{array}{l}\text { Fratura do ilio esquerdo e disjunção sacro-iliaca } \\
\text { direita }\end{array}$ & $\begin{array}{l}\text { Placa com parafusos e pino trans-iliaco com } \\
\text { parafuso auto- atarraxante. }\end{array}$ \\
\hline $\begin{array}{l}\mathrm{N}^{\circ} 07 \text {, macho, SRD, } \\
\text { 3anos, } 11 \mathrm{Kg}\end{array}$ & A. A. & Fratura bilateral de ilio & $\begin{array}{l}\text { Parafuso com PMMA em ilio direito e placa } \\
\text { com parafuso no corpo do ilio esquerdo }\end{array}$ \\
\hline $\begin{array}{l}\mathrm{N} \cdot 08, \text { macho, Dash, } 5 \\
\text { anos, } 6,1 \mathrm{Kg}\end{array}$ & A. A. & $\begin{array}{l}\text { Fratura obliqua de ilio esquerdo, asa isquiática } \\
\text { direita e cominutiva do púbis. }\end{array}$ & Placa com parafuso no corpo do itio. \\
\hline $\begin{array}{l}\text { No } 09 \text {, fêmea, SRD, } 4 \\
\text { anos, } 8,05 \mathrm{~kg}\end{array}$ & A. A. & Fratura pélvica cominutiva bilateral & $\begin{array}{l}\text { Parafusos auto- atarraxantes, fio de aço } \\
\text { em banda de tensão e PMMA em } \\
\text { acetábulo, Pinos externos no îio }\end{array}$ \\
\hline $\begin{array}{l}\mathrm{N} \cdot 10, \text { fèmea, } \mathrm{SRD}, 6 \\
\text { meses, } 11,7 \mathrm{Kg}\end{array}$ & A. A. & $\begin{array}{l}\text { Fratura do } 1 / 3 \text { distal do acetábulo esquerdo, } \\
\text { obliqua da asa do ilio esquerdo e disjunção } \\
\text { sacro-iliaca esquerda. Como também fratura de } \\
\text { fêmur e tibia do membro esquerdo. }\end{array}$ & $\begin{array}{l}\text { Parafuso ilio-sacral esquerdo. } \\
\text { Pino intramedular em fêmur e fixador } \\
\text { externo em tibia. }\end{array}$ \\
\hline $\begin{array}{l}\mathrm{N} \cdot 11, \text { fèmea, } \mathrm{SRD}, 2 \\
\text { anos, } 12 \mathrm{Kg}\end{array}$ & A. A. & $\begin{array}{l}\text { Disjunção sacro-iliaca direita e fratura do iko } \\
\text { esquerdo }\end{array}$ & Parafuso com PMMA no corpo do ilio \\
\hline
\end{tabular}

A. A.= Acidente Automobilístico; PMMA= Polimetilmetacrilato. 
Tabela 4. Dados dos pacientes com fratura pélvica submetidos ao tratamento conservativo contendo resenha, etiologia, ocorrências, osso envolvido e tipo de fratura

\begin{tabular}{|c|c|c|c|}
\hline Dados dos Pacientes & $\begin{array}{l}\text { Causa da } \\
\text { Fratura }\end{array}$ & Ocorrências & Osso/Tipo de fratura \\
\hline $\begin{array}{l}\text { № } 12 \text {, fêmea, Boxer, } 3 \\
\text { meses, } 10 \mathrm{Kg}\end{array}$ & Queda & $\begin{array}{l}\text { Cirurgia para liberação do nervo } \\
\text { isquiático }\end{array}$ & $\begin{array}{l}\text { Fratura cominutiva de ilio e acetábulo do } \\
\text { lado } \mathrm{E}\end{array}$ \\
\hline $\begin{array}{l}\mathrm{N}^{\circ} 13, \text { macho, SRD, } 6 \\
\text { anos, } 15 \mathrm{Kg}\end{array}$ & A. A. & $\begin{array}{l}\text { Óbito em consequência ao } \\
\text { politraumatismo }\end{array}$ & Fratura de ileo $\mathrm{E}$, acetábulo e púbis $\mathrm{D}$. \\
\hline $\begin{array}{l}\text { No 14, macho, Poodle, } 8 \\
\text { anos, } 4,61 \mathrm{Kg}\end{array}$ & Queda & $\begin{array}{l}\text { Recuperação total sem intervenção } \\
\text { cinúrgica }\end{array}$ & Fratura do isquio e púbis \\
\hline $\begin{array}{l}N^{\circ} 15, \text { macho, Basset, } 4 \\
\text { anos, } 6,7 \mathrm{Kg}\end{array}$ & Queda & $\begin{array}{l}\text { Colotomia para remoção de } \\
\text { fecaloma, consequente de grave } \\
\text { estreitamento }\end{array}$ & $\begin{array}{l}\text { Fratura de asa do ilio } \mathrm{E} \text { e bilateral do } \\
\text { isquio. }\end{array}$ \\
\hline $\begin{array}{l}\mathrm{N}^{\circ} 16, \text { macho, SRD, } 1,5 \\
\text { anos, } 8,83 \mathrm{Kg}\end{array}$ & A. A. & $\begin{array}{l}\text { Óbito em consequência ao } \\
\text { politraumatismo, ruptura de uretra e } \\
\text { bexiga. }\end{array}$ & Fratura de ilio, isquio e púbis $\mathrm{E}$ \\
\hline $\begin{array}{l}\text { No } 17 \text {, macho, Pinscher, } 8 \\
\text { meses, } 3 \mathrm{Kg}\end{array}$ & A. A. & $\begin{array}{l}\text { Óbito - fistula anal, ruptura de } \\
\text { cólon. TCE Grave. }\end{array}$ & $\begin{array}{l}\text { Fratura de ilio } \mathrm{E} \text {, fratura do acetábulo } \mathrm{E} \text {, } \\
\text { fratura do púbis bilateral e fratura do } \\
\text { isquio } \mathrm{E} \text {. }\end{array}$ \\
\hline $\begin{array}{l}N^{\circ} 18, \text { fêmea, Poodle, } 3 \\
\text { anos, } 6 \mathrm{~kg} \text {. }\end{array}$ & A. A. & $\begin{array}{l}\text { Óbito, em consequência de } \\
\text { Mielomalacia hemorrágica (Fratura } \\
\text { em T10) }\end{array}$ & $\begin{array}{l}\text { Fratura de acetábulo } \mathrm{D} \text {, huxação } \\
\text { coxofemoral } \mathrm{E} \text {, fratura de isquio } \mathrm{E} \text {, } \\
\text { fratura de púbis. }\end{array}$ \\
\hline $\begin{array}{l}N^{\circ} 19, \text { fèmea, SRD, } \\
7 \text { meses, } 7 \mathrm{~kg}\end{array}$ & A. A. & $\begin{array}{l}\text { Correção cirúrgica para ruptura } \\
\text { diafragmática e correção cinúrgica } \\
\text { para fratura de coluna lombar }\end{array}$ & $\begin{array}{l}\text { Luxação sacro-iliaca D, huxação coxo- } \\
\text { femoral com fratura acetabular D, } \\
\text { Fratura de vértebra lombar. }\end{array}$ \\
\hline $\begin{array}{l}\text { No20, macho, SRD, } 14 \mathrm{~kg} \\
9 \text { meses. }\end{array}$ & A. A & $\begin{array}{l}\text { Óbito em consequência ao } \\
\text { politraumatismo e ruptura de cólon } \\
\text { descendente. }\end{array}$ & Fratura de ilio, acetábulo e púbis $\mathrm{D}$. \\
\hline
\end{tabular}

A. A. = Acidente Automobilístico; D: direito, E; esquerdo; TCE: trauma crânio encefálico.

Os exames clínicos e ortopédicos detalhados permitiram predizer, através dos achados, a presença de risco de morte, local e intensidade das lesões, para, posteriormente, se realizarem os exames radiográficos e confirmar as suspeitas.

Através do estudo das radiografias observouse que em nenhum caso houve fratura de um único osso da pelve, fato que concorda com o que relatam HENRY (1985), DECAMP (1992), HOULTON \& DYCE (1994), OLMSTEAD \& MATIS (1998), PIERMATTE et al. (2006) e HARASEN (2007). Em $80 \%$ dos casos (16 animais) houve fratura bilateral da estrutura pélvica, $17(85 \%)$ dos animais apresentaram fratura ilíaca e, desses, quatro apresentaram transtornos bilaterais. Em relação à linha de fratura no corpo do ílio, em 10 pacientes (50\% do total) era oblíqua, em seis $(30 \%)$ foi transversa e só um animal fraturou a asa do ílio $(5 \%)$. Oito animais (40\%) tiveram fratura isquiática, sendo bilaterais em apenas dois pacientes, sete $(35 \%)$ apresentaram fratura púbica, sendo também bilateral em dois. Do total de pacientes com trauma pélvico, quatro (20\%) tiveram disjunção sacroilíaca.

A alta taxa de fraturas ilíacas encontradas está de acordo com o que descrevem HENRY (1985) e HARASEN (2007), quando afirmam que a fratura de ílio é a mais comum das fraturas pélvicas. Essas observações têm importância por apontarem o envolvimento das estruturas pelas quais incidem as cargas advindas do membro pélvico para o eixo espinhal em $95 \%$ das fraturas pélvicas por eles analisadas.

Dependendo da intensidade da força mecânica e da conformação das fraturas pélvicas, surgem índices significantes de morbidade $\mathrm{e}$ mortalidade, em associação a injúrias nos outros órgãos (DECAMP, 1992; HOULTON \& DYCE, 1994; INNES \& BUTTERWORTH, 1996), como foi observado nos animais desta pesquisa (Figura 2). Segundo NEWTON (1985), aproximadamente 8\% dos animais tem injúrias em outros órgãos, sendo comum envolvimento de bexiga, uretra, reto, nervo periférico, tendão pré-púbico e lacerações na região perineal. Nos pacientes 16 e 17, respectivamente, houve ruptura de uretra e de cólon com formação de fístula perianal; em consequêcia, ambos vieram a óbito. 


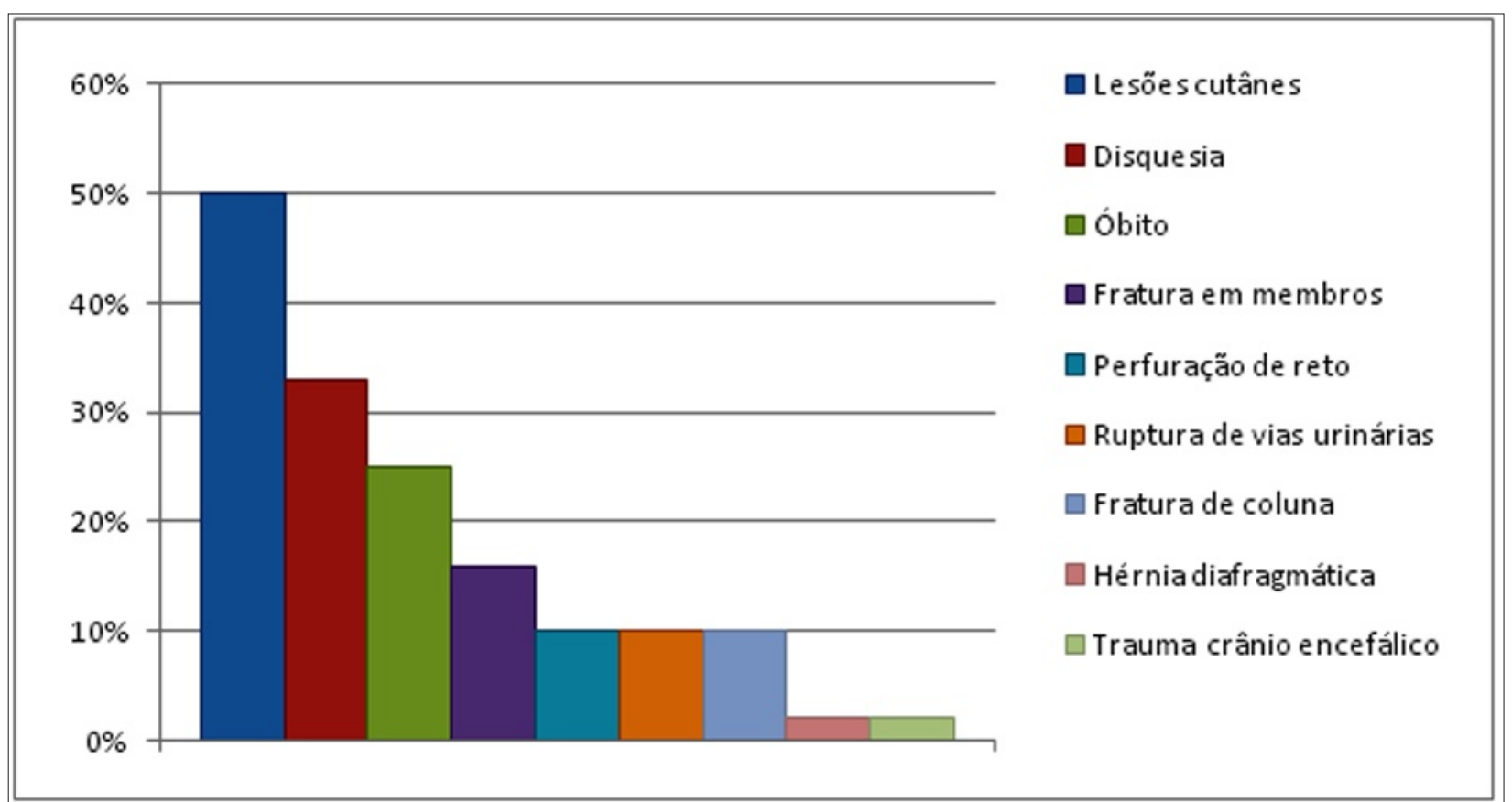

Figura 2. Distribuição dos transtornos concomitantes ou presentes nos 20 cães com fratura pélvica.

Podem ainda ocorrer nos cães com fratura pélvica outros problemas ortopédicos ou espinhais (EDWARDS, 1993; DENNY \& BUTTERWORTH, 2006), como fraturas e luxações, observadas na décima vértebra torácica (T10) do paciente 18 e na sexta vértebra lombar (L6) do paciente 19. O primeiro desenvolveu mielomalacia hemorrágica e o outro voltou a deambular normalmente após laminectomia. Ocorreram também fraturas em membros nos pacientes 5 e 10 , sendo, respectivamente, de fêmur e de fêmur e tíbia, que foram reparadas cirurgicamente.

Ainda foram observadas lesões cutâneas na região dos membros, região abdominal e genitália externa em $50 \%$ dos pacientes, como também a presença de hematomas e edema em $22 \%$ dos animais.

Fraturas da pelve podem lacerar o nervo isquiático e femoral. O diagnóstico de neuropatia do nervo isquiático foi fundamentado no histórico e nos sinais clínicos (WALKER, 1981; BRAUND, 2003; FORTERRE et al., 2007) e associado à fratura por ser ipsilateral à lesão. Em três pacientes foi observado déficit de nervo isquiático (pacientes 11, 12 e 17), sendo a etiologia diferente, porém, a manifestação clínica a mesma. No primeiro, a neuropraxia ocorreu durante o procedimento para osteossíntese de fratura ilíaca, sendo que os déficits neurológicos foram abordados conservativamente e resultaram na resolução dos mesmos após 90 dias.
No segundo caso, os sinais neurológicos eram decorrentes da compressão nervosa progressiva devido à proliferação de tecido fibroso (DEWEY, 2006), em consequência à lesão acetabular que não foi identificada e, por isso, tratada conservativamente. Alteração semelhante foi descrita por FEARNSIDE \& BLACK (1999) em um cão com fratura de acetábulo tratado de forma conservadora cinco meses antes do aparecimento dos sinais clínicos. Frente aos dados, optou-se no cão 12 pela neurólise cirúrgica que resultou, após 60 dias, na recuperação total dos reflexos e funções perdidas. JACOBSON \& SCHRADER (1987) relataram uma recuperação variando de adequada a excelente em $81 \%$ dos animais com fratura pélvica em até 16 semanas após diagnóstico da neuropraxia.

No último desses três pacientes, observou-se ausência de resposta aos testes do nervo isquiático esquerdo, estando preservados os reflexos nos demais nervos e membros, além de o animal apresentar cegueira central, estrabismo divergente e miose bilateral arresponsiva, devido ao concomitante traumatismo crânio-encefálico. Além das lesões nervosas e ósseas, houve fístula perianal, com suspeita de ruptura de reto ou cólon. Embora a perfuração retal seja incomum, é imperativo o diagnóstico precoce, uma vez que a demora no tratamento tornará o prognóstico ruim (DENNY \& BUTTERWORTH, 2006).

Dentre os 20 pacientes, sete apresentaram, no 
atendimento, disquesia ou obstipação, fato decorrente da estenose do canal pélvico e da dor. Desses, quatro foram operados, resultando na reconstrução do canal e redução da dor, normalizando a evacuação. Dois desses sete pacientes foram tratados de forma conservativa com a utilização de laxantes orais, repouso e analgésicos. O caso 15 se encontrava há 15 dias sem defecar, devido ao estreitamento do canal pélvico pelo processo cicatricial de uma fratura pélvica não operada, como citado por NEWTON (1996), necessitando de colotomia, laxantes e adequação da dieta.

EDWARDS (1993) afirma que o tratamento cirúrgico ajuda na redução da morbidade e mortalidade relacionada às fraturas, além de encurtar o período de cuidados hospitalares, complicações e recuperação da mobilidade. Nos pacientes operados houve retorno a deambulação, observada precocemente, graus 3 e 4 de claudicação em nove pacientes, após sete dias da cirurgia, evoluindo para graus 4 e 5 em dez pacientes após 60 dias e a recuperação total da função ortopédica foi verificada após 90 dias em todos os operados. Resultado que não se repetiu nos pacientes tratados conservativamente, sendo observada claudicação persistente de grau 3 e 4 em dois dos pacientes após 90 dias. Acredita-se que a estabilização cirúrgica dos segmentos ósseos é responsável por conferir redução da dor, com uma diminuição evidente dessa em $100 \%$ dos pacientes avaliados no momento précirúrgico com grau 0 e 1, para os graus 2 e 3 . Também em $81 \%$ dos pacientes, após sete dias, elevou-se a qualidade de vida e reduziram-se as necessidades de enfermagem, enquanto $55 \%$ dos não operados vieram a óbito, dois apresentaram claudicação persistente grau 2 e os dois restantes tiveram que ser operados para corrigir complicações (obstipação e neuropraxia) em consequência da falta de tratamento prévio adequado, com recuperação parcial, sendo ainda observadas constipação e claudicação.

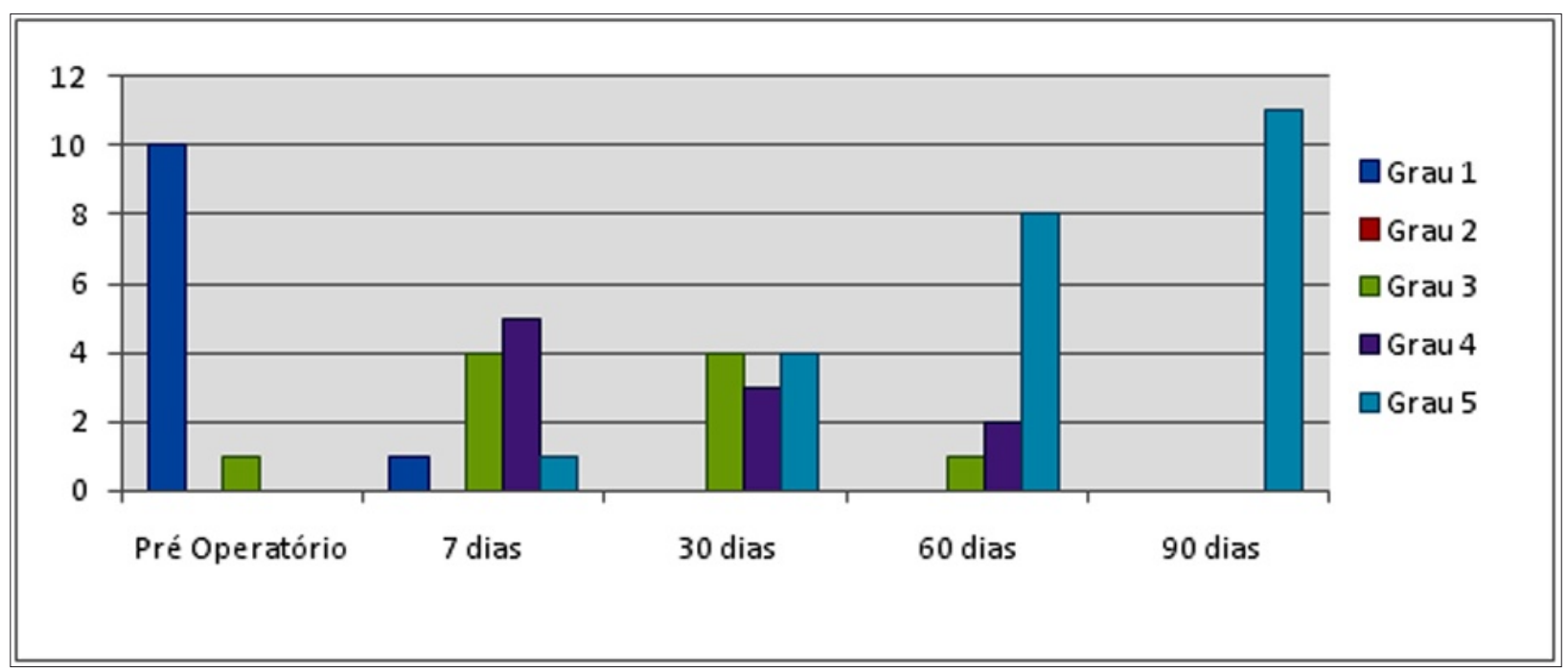

Figura 3 Avaliação do escore de claudicação dos pacientes com fratura de pelve submetida a procedimento cirúrgico.

Segundo OLMSTED (1995), ocorrem mais casos de fraturas ilíacas e acetabulares que luxações sacro-ilíacas, fato observado, visto que a maioria dos casos possuía fratura de ílio (19) associado a outras fraturas, de púbis (10), ísquio (8), acetabulares (4) e disjunção sacro-ilíaca. Essa última ocorreu em quatro de 20 pacientes, associada à fratura do ílio contralateral. Desses animais, três fizeram tratamento cirúrgico através da colocação de um parafuso de compressão que atravessava o ílio e se fixava no sacro, conforme recomendado por

\section{PIERMATTEI et al. (2006).}

Nos animais em que o tratamento foi cirúrgico, em 4/11 (36\%) utilizou-se placa com parafusos e em 5/11 (45\%), parafusos interligados com PMMA. No caso 7, 1/11 (9\%), com fratura ilíaca bilateral, foi empregado no ílio direito parafuso unido com PMMA e no esquerdo, placa com parafuso. Já no caso 10, utilizou-se um parafuso (efeito compressivo) para redução de disjunção sacro-ilíaca. Nesses animais não se observou diferença quanto à recuperação ou 
dificuldades quanto ao procedimento cirúrgico; muito embora a placa tenha vantagens, já que, devido à sua rigidez, ela confere (após sua fixação no segmento ilíaco caudal) maior facilidade no alinhamento e redução da fratura, quando comparada com o PMMA.

\section{CONCLUSÃO}

Os resultados observados assinalam que em cães com trauma pélvico deve-se procurar primariamente a resolução cirúrgica e a presença de outras lesões orgânicas concomitantes incapacitantes ou com potencial de induzir ao óbito.

\section{AGRADECIMENTOS}

Ao $\mathrm{CNPq}$ pela bolsa de mestrado, e à FACEPE pela bolsa de PIBIC.

\section{REFERÊNCIAS}

BONATH, K.H.; PRIEUR, W.D. Kleintierkrankheiten: Orthopädische Chirurgie und Traumatologie. Stuttgart: Ulmer, 1998, p.576-588.

BRAUND, K.G. Traumatic Neurophaty. In: Braund's Clinical Neurology in Small Animals: Localization, Diagnosis and Treatment, Vite, C. H., International Veterinary Information Service, Ithaca, NY (www.ivis.org), 2003. http://www.ivis.org/advances/Vite/braund28/chapter_frm. asp?LA=1. Acesso em abril 2008.

DECAMP, C.E. Principles of pelvic fracture management. Seminars in veterinary medicine and surgery. v.7, n.1, p.63-70, 1992.

DENNY, H.R.; BUTTERWORTH, S.J. Pelve in Cirurgia Ortopédica em Cães e Gatos, 4.ed. São Paulo: Roca, 2006. cap.39, p.341-351.

DEWEY, C.W. Doenças do Sistema Nervoso Periférico: Mononeuropatias e Polineuropatias. In: DEWEY, C. W. Neurologia de Cães e Gatos. São Paulo: Roca, 2006. p.215-239.

DOYLE, N.D. Rehabilitation of fractures in small animals: maximize outcomes; minimize complications Clinical techniques in small animal practice. v.19, n.3, p.180-191, 2004.

EDWARDS, K.P. Orthopedic trauma: pelvic fracture. Todays OR Nurse. v.15, n.4, p.24-28, 1993.

FEARNSIDE, S.M.; BLACK, A.P. Delayed onset of sciatic neuropathy following coxofemoral joint fracture. Australian veterinary journal. v.29, n.2, p.63-68, 1999.
FORTERRE, F.; TOMEK, A.; RYTZ, U.; BRUNNBERG, L.; JAGGY, A.; SPRENG, D. Iatrogenic Sciatic Nerve Injury in Eighteen Dogs and Nine Cats (1997-2006). Veterinary Surgery. v.36, p. 464-471, 2007.

HARASEN, B. Pelvic fractures. The Canadian veterinary journal. v.48, n.4, p.427-428, 2007.

HENRY, W.B. A method of bone plating for repairing iliac and acetabular fractures. The Compendium on continuing education. v.7, n.11, p.924-938, 1985.

HOULTON, J.; DYCE, J. Tratamiento de las fracturas pélvicas em perros y gatos. Waltham Focus. v.4, n.2, p.17-25, 1994.

INNES, B; BUTTERWORTH, S. Decision making in the treatment of pelvic fractures in small animals. In Practice, London, v.18, n. 5, p.215-221, 1996.

JACOBSON A, SCHRADER S.C. Peripheral nerve injury associated with fracture or fracture-dislocation of the pelvis in dogs and cats: 34 cases (1978-1982).

Journal of the American Veterinary Medical Association. v.190, n.5, p.569-572, 1987.

JOHNSON, A.L. Pelvic Fractures In: FOSSUM, T.W. Small Animal Surgery, 3.ed, St. Louis: Mosby, 2007. p.1087-1102.

KEMPER, B; SEVERO, M.S; FIGUIREDO, M.L; SILVA, A.A; CHIORATTO, R; MARQUES, N; TUDURY, E.A. Fixação percutânea externa complementar na osteossíntese de fratura pélvica cominutiva bilateral- relato de caso em um cão. Clínica Veterinária. n.74, p.40-44, 2008.

KNAP, K.; JOHNSON, A.J.; SCHULZ, K. Fundamentals of Physical Rehabilitation, in: FOSSUM, T.W. Small Animal Surgery, 3ed. St. Louis: Mosby, 2007, p.111129.

LASCELES, S.J.; BUTTERWOR, T. H.; WATERMAN, A. E. Postoperative analgesic and sedative effects of caprofen and phetidine in dogs. Veterinary Record. v.134, p. 187-190, 1994.

LENEHAN, T.M.; TARVIN, G.B. Fractures of the hind limb. In: BOJRAB, M.J. Current Techniques in Small Animal Surgery. 4.ed, Philadelphia: Williams \& Wilkins. 1998. p.1033-1040.

NEWTON, C.D. Fracture Repair. In: LIPOWITZ, A.L.; CAYWOOD, D.D.; NEWTON, C.D.; SCHWARTZ, A. Complications in Small Animal Surgery. Philadelphia: Williams\&Wilkins. 1996. p.563-597.

NEWTON, C.D. Fractures of the Pelvis. In: NEWTON, C.D; NUNAMAKER, D.M. Textbook of Small Animal Orthopedics. Philadelphia: Lipencott, 1985. p.393-402.

OLMSTEAD, M.L. Fractures of the Bones of the Hind Limb. In: OLMSTEAD, M.L. Small Animal Orthopedics, St. Louis: Mosby, 1995. p.219-228. 
OLMSTEAD, M.L. The Pelvis and Sacroiliac Joint. In: COUGHLAN, A.; MILLER, A. Manual of Small Animal Fracture Repair and Management, Hampshire: BSAVA, 1998. p.217-228.

OLMSTEAD, M.L.; MATIS, U. Fractures of the Pelvis. In: BRINKER, W.O.; OLMSTEAD, M.L.; SUMNERSMITH, G.; PRIEUR, W.D. Manual of Internal Fixation in Small Animal. Berlin: Springer, 1998. p.148154.

PAYNE, J.T. Selecting a method for managing pelvic fractures in dogs and cats. The Veterinary Record. v.88, n.10, p.969-973, 1993.

PIERMATTEI, D.L.; FLO, G.L.; DECAMP, C.E. Fractures of the Pelvis. In: Small Animal Orthopedics and fracture Repair. 4.ed, St Louis: Saunders, 2006. p.433-460.

PIERMATTEI, D.L.; JOHNSON, K.A. Surgical Approaches to the Bones and Joints of the Dog and
Cat. 4ed, Philadelphia: Saunders, 2004, 400p.

ROEHSIG, C.; ROCHA, L.B.; BARAÚNA JÚNIOR, D.; CHIORATTO, R.; MELO E SILVA, S.R.A.; KEMPER, B.; ARAÚJO, F.P.; ALMEIDA, A.C.M.; TUDURI, E.A. Fixação de fraturas ilíacas em cães com parafusos, fios de aço e cimento ósseo de polimetilmetacrilato. Ciência Rural, v.38, n.6, p. 1675-1681, 2008

TOMLINSON, J.L. Fractures of the Pelvis. In: SLATTER, D.H. Text Book of Small Animal Surgery. 3ed, Philadelphia: Saunders, 2003. p.1989-2001.

TUDURY, E. A.; RAISER, A.G. Redução de fraturas distais de fêmur de cães empregando pinos de Steinmann em substituição aos de Rush. Revista do Centro de Ciências Rurais. v.15, n.2, p.1414-155, 1985.

WHITTICK, W G, Canine Orthopedics, Philadelphia: Lea \& Febiger, 1978.

Protocolado em: 27 jul. 2008. Aceito em: 11 abr. 201. 